\title{
Quiet T1-Weighted Pointwise Encoding Time Reduction with Radial Acquisition for Assessing Myelination in the Pediatric Brain
}

\author{
(D) N. Aida, (D) Tiwa, (D). Fujii, (D). Nozawa, (D). Enokizono, (D). Murata, and (D) T. Obata
}

\begin{abstract}
BACKGROUND AND PURPOSE: T1-weighted pointwise encoding time reduction with radial acquisition (PETRA) sequences require limited gradient activity and allow quiet scanning. We aimed to assess the usefulness of PETRA in pediatric brain imaging.
\end{abstract}

MATERIALS AND METHODS: We included consecutive pediatric patients who underwent both MPRAGE and PETRA. The contrast-tonoise and contrast ratios between WM and GM were compared in the cerebellar WM, internal capsule, and corpus callosum. The degree of myelination was rated by using 4-point scales at each of these locations plus the subcortical WM in the anterior frontal, anterior temporal, and posterior occipital lobes. Two radiologists made all assessments, and the intra- and interrater agreement was calculated by using intraclass correlation coefficients. Acoustic noise on MPRAGE and PETRA was measured.

RESULTS: We included 56 patients 5 days to 14 years of age (mean age, 36.6 months) who underwent both MPRAGE and PETRA. The contrast-to-noise and contrast ratios for PETRA were significantly higher than those for MPRAGE $(P<.05)$, excluding the signal ratio for cerebellar WM. Excellent intra- and interrater agreement were obtained for myelination at all locations except the cerebellar WM. The acoustic noise on PETRA (58.2 dB[A]) was much lower than that on MPRAGE (87.4 dB[A]).

CONCLUSIONS: PETRA generally showed better objective imaging quality without a difference in subjective image-quality evaluation and produced much less acoustic noise compared with MPRAGE. We conclude that PETRA can substitute for MPRAGE in pediatric brain imaging.

ABBREVIATIONS: $C C=$ corpus callosum; ICC = intraclass correlation coefficient; PETRA = pointwise encoding time reduction with radial acquisition

M R imaging is widely used for brain assessment in both adults and children, enabling the noninvasive and detailed evaluation of morphologic and functional abnormalities. ${ }^{1,2}$ However, MR imaging has some drawbacks. Of note, an average scanning time of 20-30 minutes is usually required for a routine brain examination, during which the patient is subjected to loud acoustic noise. Consequently, the application of MR imaging is limited in infants and small children, who often need sedation to undergo MR imaging. ${ }^{3-5}$ Even under sedation, the acoustic noise from MR

Received October 30, 2015; accepted after revision January 20, 2016.

From the Department of Radiology (N.A., T.N., Y.F., K.N., M.E.), Kanagawa Children's Medical Center, Yokohama, Japan; Department of Radiology (T.N.), Tokai University School of Medicine, Isehara, Japan; Siemens Healthcare K.K. (K.M.), Ohsaki, Tokyo, Japan; and Research Center for Charged Particle Therapy (T.O.), National Institute of Radiological Sciences, Chiba, Japan.

Please address correspondence to Tetsu Niwa, MD, PhD, Department of Radiology, Tokai University School of Medicine, 143 Shimokasuya, Isehara, 259-1193, Japan; e-mail: niwat@tokai-u.jp

三 Indicates article with supplemental on-line table.

http://dx.doi.org/10.3174/ajnr.A4747 imaging can make children restless or cause them to awaken, resulting in severe motion artifacts or incomplete examinations.

With the increased use of MR imaging in children, it is important to reduce the loudness of MR imaging scanners to ensure that scans are completed with minimal distress to the child and minimal artifacts on the acquired images. Because the acoustic noise of MR imaging is produced by the vibration of gradient coils during the scan, noise reduction can be achieved by decreasing the noise from these coils. One such method involves sealing gradient coils in a vacuum chamber. ${ }^{6}$ More recently, several methods have been introduced to reduce acoustic noise that do not involve altering the scanner hardware. These techniques include the use of acoustically optimized pulse shapes of the gradient coils to cancel single frequencies extended by a second frequency, ${ }^{7}$ ultrashort TE sequences such as zero TE, ${ }^{8}$ sweep imaging with Fourier transformation, ${ }^{9}$ and pointwise encoding time reduction with radial acquisition (PETRA).${ }^{10}$ Of these, PETRA requires limited gradient activity, which creates a particularly quiet MR imaging scan. ${ }^{10}$ Considering that quiet sequences should be useful for reducing patient stress during the scan, this technique might particularly 


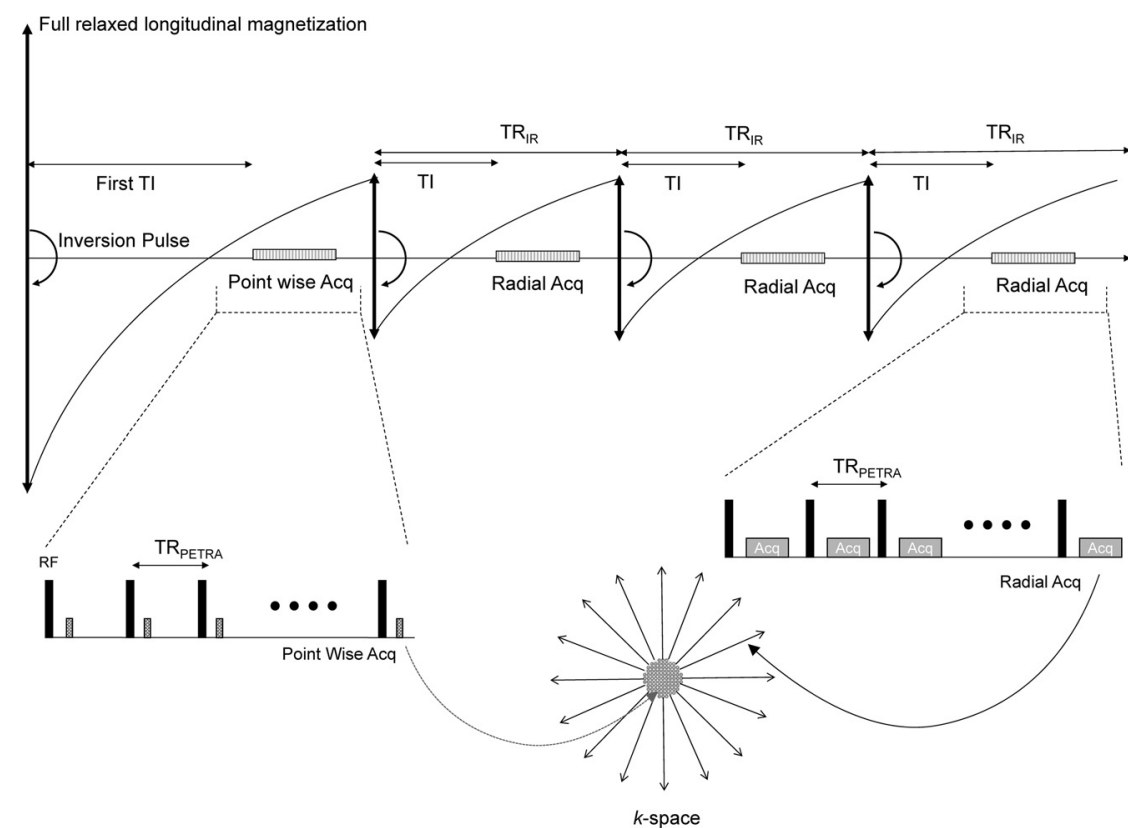

FIG 1. Pulse sequence diagram for the inversion pulse of the PETRA sequence. The center part of the $k$-space is acquired with pointwise encoding after the first inversion pulse, and the outer edge of the volumetric $k$-space is acquired with radial projections after the second and later inversion pulses. Acq indicates acquisition.

benefit children. PETRA sequencing uses an inversion recovery pulse to yield T1WI, which is a basic MR imaging sequence that can be used to assess myelination in children.

In this study, we therefore aimed to compare the measurements of pediatric brain myelination obtained by using a quiet T1-weighted PETRA sequence with those captured by MPRAGE to assess the suitability of PETRA for pediatric brain imaging.

\section{MATERIALS AND METHODS \\ Patients}

We performed T1-weighted PETRA in addition to routine MR imaging in all pediatric patients undergoing brain MR imaging with sedation from May to October 2013. To avoid unnecessary delays and to keep scanning times short, we did not perform PETRA for emergency cases and we excluded older children who did not require sedation. We added T1-weighted PETRA after routine MR imaging examinations without additional sedation for consecutive patients, excluding cases with severe motion artifacts. Our institutional review board (Kanagawa Children's Medical Center) approved this prospective study, and written consent was obtained from the parents.

\section{MR Imaging}

All MR imaging examinations were performed on a 3T clinical scanner (Magnetom Verio; Siemens, Erlangen, Germany) by using a 32channel head coil. The routine MR imaging brain sequence included axial and coronal T2-weighted fast spin-echo, axial MPRAGE, and axial DWI. In addition, T1-weighted PETRA was performed.

PETRA uses an ultrashort TE sequence that acquires $k$-space data in a combination of the central and outer part of the $k$-space during different processes. ${ }^{10-12}$ Particularly, the central part of the $k$-space is acquired with pointwise encoding after the first inversion pulse, whereas the outer edge of volumetric $k$-space is acquired by using radial projections after the second and later inversion pulses (Fig 1). PETRA keeps gradients invariable for almost an entire repetition, and the gradients change only slightly at the end of each repetition. As a result, PETRA achieves minimal vibration of the gradient coils, generating only residual acoustic noise because of radiofrequency switching.

In this study, we adjusted PETRA sequences for clinical use with children. First, PETRA was set to have the shortest possible acquisition time while maintaining sufficient spatial resolution. Second, the first TI on PETRA was individualized to obtain an appropriate contrast for the pediatric brain. The first TI might greatly affect the contrast of the PETRA image because the central part of the $k$-space is acquired with a first inversion pulse in the PETRA sequence (Fig 1) and the $\mathrm{T} 1$ value of the brain is known to be longer in infants and small children than in older children and adults. ${ }^{13,14}$ Therefore, the first TI was set to a longer value than that used in adult scans. We measured the T1 value of each child before PETRA sequencing by using double flip angle FLASH sequences with the following parameters: $\mathrm{TR} / \mathrm{TE}=10 / 1.37 \mathrm{~ms}$; double flip angle $=3^{\circ}, 19^{\circ} ; \mathrm{FOV}=230 \mathrm{~mm}$; matrix $=95 \times 128$; thickness $=4 \mathrm{~mm}$; number of sections $=19$; acquisition time $=26$ seconds.

A T1 map was subsequently generated by the MR imaging scanner software. We placed an ROI that corresponded to the contour of the brain parenchyma at the level of the basal ganglia on the T1 map, and the T1 value of the brain parenchyma was measured, excluding values of $\geq 3000 \mathrm{~ms}$ to remove the CSF space. Then, the first TI on PETRA was set as the measured T1 value multiplied by 0.7 , and we set the upper and lower limits of the first TI at 1000 and $1800 \mathrm{~ms}$, respectively; if the measured T1 value exceeded these limits, the first TI was set at 1000 or $1800 \mathrm{~ms}$, respectively. This method was determined by a pilot study and a study with adult volunteers (N.A., unpublished data, April 2013). The imaging parameters for PETRA and MPRAGE are shown in Table 1. The actual MPRAGE parameters varied depending on the child's head size.

\section{Image Analysis}

PETRA images were reformatted in the axial plane in the same section angulation and thickness as those for MPRAGE. Images were assessed qualitatively and quantitatively.

\section{Quantitative Analysis}

Quantitative analysis was performed by assessing the imaging contrast and calculating the contrast-to-noise and signal ratios between WM and GM on MPRAGE and PETRA. The signal intensity and SD at the WM and adjacent GM were measured by a neuroradiologist (M.E., with 8 years of experience in neuroradiology) by ROI placement. This analysis was conducted by selecting a population with relatively advanced myelination (ie, children older than 7 months of 


\begin{tabular}{|c|c|c|c|c|}
\hline \multirow[b]{2}{*}{ Parameters } & \multicolumn{3}{|c|}{ MPRAGE } & \multirow[b]{2}{*}{ PETRA } \\
\hline & Small FOV & Middle FOV & Large FOV & \\
\hline FOV (mm) & 150 & 200 & 240 & 285 \\
\hline Orientation & Axial & Axial & Axial & Sagittal \\
\hline First TI (ms) & NA & NA & NA & 1000-1800 \\
\hline $\mathrm{TI}(\mathrm{ms})$ & 800 & 800 & 800 & 700 \\
\hline TR (ms) & 1570 & 1570 & 1570 & 3.75 \\
\hline $\mathrm{TE}(\mathrm{ms})$ & 2.14 & 2.79 & 2.77 & 0.07 \\
\hline Echo space (ms) & 5.2 & 5.2 & 5.2 & 3.75 \\
\hline Flip angle (degrees) & 9 & 9 & 9 & 6 \\
\hline Section thickness (ms) & 1.0 & 1.0 & 1.0 & 0.8 \\
\hline Section oversampling (\%) & 100 & 36.4 & 25.0 & 0 \\
\hline Matrix & $154 \times 192$ & $168 \times 192$ & $224 \times 256$ & $352 \times 352$ \\
\hline Radial spokes & NA & NA & NA & 35,000 \\
\hline GRAPPA & 2 & 2 & 2 & NA \\
\hline Resolution (mm) & $0.98 \times 0.78 \times 1.00$ & $1.04 \times 1.04 \times 1.00$ & $0.94 \times 0.94 \times 1.00$ & $0.81 \times 0.81 \times 0.81$ \\
\hline Scan time (min/sec) & $3: 27$ & $3: 05$ & $3: 05$ & $4: 20$ \\
\hline
\end{tabular}

Note:-NA indicates not available; GRAPPA, generalized autocalibrating partially parallel acquisition.

Table 2: White-gray matter contrast for MPRAGE and PETRA

\begin{tabular}{|c|c|c|c|c|c|c|}
\hline \multirow[b]{2}{*}{ Assessed Location } & \multicolumn{3}{|c|}{ Contrast-to-Noise Ratio } & \multicolumn{3}{|c|}{ Contrast Ratio } \\
\hline & MPRAGE & PETRA & $P$ Value & MPRAGE & PETRA & $P$ Value \\
\hline Cerebellar white matter & $4.02 \pm 0.78$ & $6.48 \pm 1.75$ & $<.001$ & $1.27 \pm 0.06$ & $1.26 \pm 0.01$ & .82 \\
\hline $\begin{array}{l}\text { Anterior part of the posterior limb } \\
\text { of the internal capsule }\end{array}$ & $2.25 \pm 0.68$ & $3.69 \pm 0.87$ & $<.001$ & $1.11 \pm 0.04$ & $1.17 \pm 0.05$ & $<.001$ \\
\hline \multicolumn{7}{|l|}{ Corpus callosum } \\
\hline Genu & $6.74 \pm 2.36$ & $8.41 \pm 3.90$ & .004 & $1.46 \pm 0.12$ & $1.59 \pm 0.20$ & $<.001$ \\
\hline Splenium & $6.01 \pm 1.87$ & $8.17 \pm 3.07$ & $<.001$ & $1.42 \pm 0.10$ & $1.45 \pm 0.12$ & $<.001$ \\
\hline
\end{tabular}

age). ROIs were placed at the following anatomic structures: the cerebellar WM and the adjacent cortex, the anterior part of the posterior limb of the internal capsule and thalami, the genu and splenium of the corpus callosum (CC), and the adjacent cerebral cortex. The contrast-to-noise and contrast ratios were calculated by using the following formulas, where SI is the signal intensity and SD is the standard deviation:

Contrast-to-Noise Ratio

$$
=\left(\text { Mean } \mathrm{SI}_{\mathrm{WM}}-\text { Mean } \mathrm{SI}_{\mathrm{GM}}\right) /\left[\left(\mathrm{SD}_{\mathrm{WM}}{ }^{2}+\mathrm{SD}_{\mathrm{GM}}{ }^{2}\right)\right]^{1 / 2}
$$

Contrast Ratio $=$ Mean $\mathrm{SI}_{\mathrm{WM}} /$ Mean $\mathrm{SI}_{\mathrm{GM}}$.

The ROI measurement was performed on both the right and left sides, except for the corpus callosum, and the calculated values were averaged.

\section{Qualitative Assessment}

Qualitative assessments were provided by experienced radiologists (K.N. and Y.F., who had 20 and 10 years of experience in pediatric neuroradiology, respectively). The radiologists independently rated the degree of myelination on MPRAGE and PETRA in a random order by using a clinical PACS viewer. The following 2 sections were used for this assessment: section 1, at the level of the inferior (temporal) horn of the lateral ventricle; and section 2, at the level of the foramina of Monro. In turn, the following anatomic locations were evaluated at each section: the subcortical WM in the anterior temporal lobe and the cerebellar WM at section 1; and the anterior part of the posterior limb of the internal capsule, genu, and splenium of the CC, and the subcortical WM in the anterior frontal and posterior occipital lobe at section 2. Be- cause myelination appears as a relative hypersignal on T1WI, the degree of the myelination was assessed by the signal intensity at each anatomic location in comparison with the adjacent GM. The following 4-point scale was used for this assessment: 0, hyposignal; 1 , isosignal; 2, slight hypersignal; and 3, prominent hypersignal.

\section{Acoustic Noise}

In addition to the patient study, we also measured acoustic noise by MPRAGE and PETRA during the scan of a phantom by using a 32-channel head coil. The acoustic noise level of each sequence was recorded by using a microphone (NL-32; Rion, Tokyo, Japan), which was placed horizontally at a distance of $2.5 \mathrm{~m}$ from the front panel of the MR imaging scanner. The measurements took $10 \mathrm{sec}-$ onds to complete, and they were repeated 16 times. The measured noise values were averaged for each sequence. For comparison, the ambient sound level was measured 8 times for 10 seconds each.

\section{Statistical Analysis}

Statistical analyses were performed by using the MedCalc software package for Windows, Version 15.4 (MedCalc Software, Mariakerke, Belgium), and we considered $P$ values $<.05$ to indicate a statistically significant difference. The contrast-to-noise and signal ratios for MPRAGE and PETRA were compared by using paired sample $t$ tests. Inter- and intrarater agreement for the myelination scores between MPRAGE and PETRA was evaluated by calculating the intraclass correlation coefficient (ICC). ICCs were interpreted by using the criteria reported by Landis and $\mathrm{Koch}^{15}$ : an ICC of $0.01-0.20$ indicated slight agreement; an ICC of 0.21-0.40, fair agreement; an ICC of $0.41-0.60$, moderate agreement; an ICC of $0.61-0.80$, substantial agreement; and an ICC of 0.81-1.0, near-perfect agreement. 


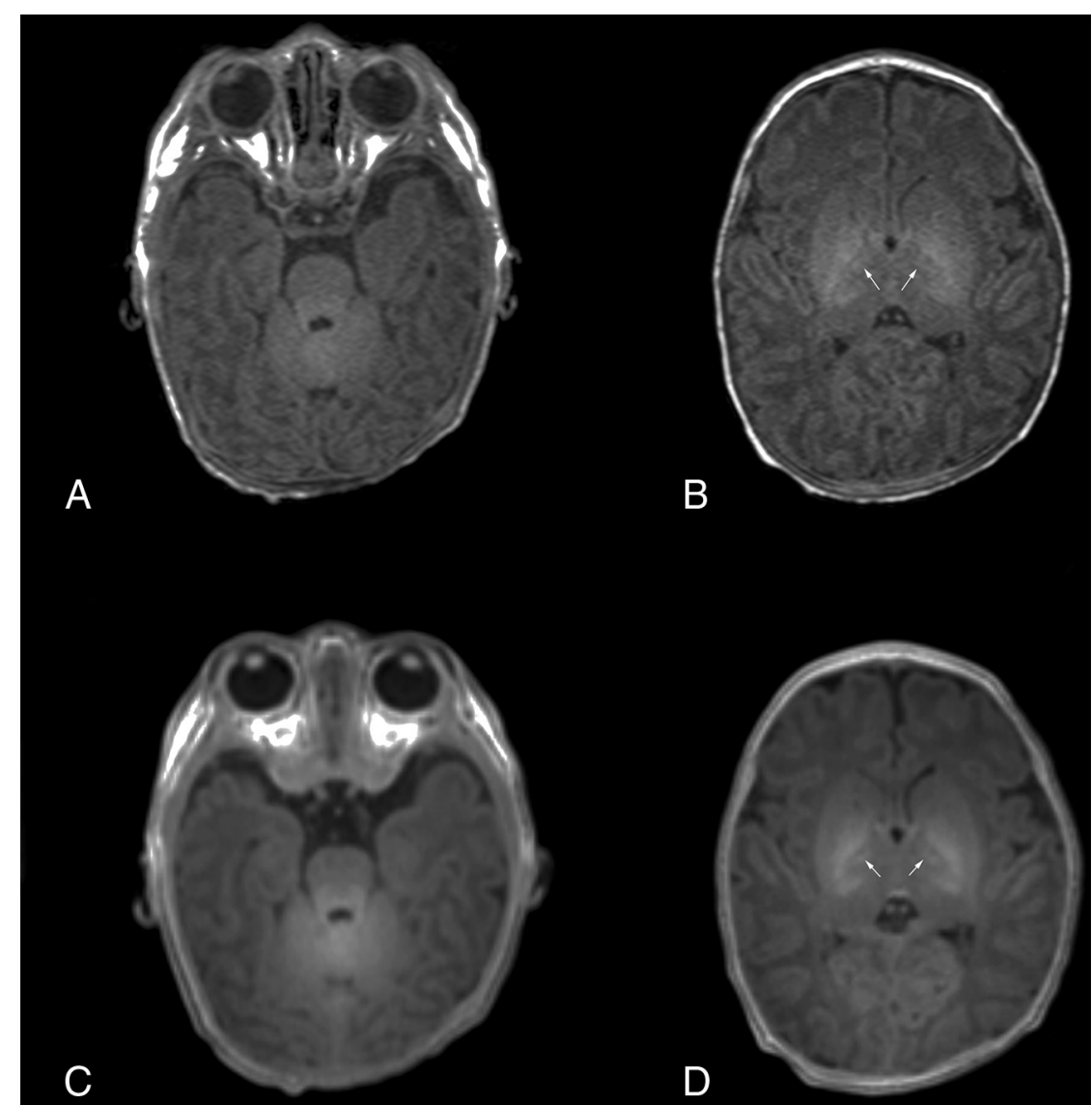

FIG 2. Images of a 9-day-old neonate. MPRAGE ( $A$ and $B$ ) and PETRA ( $C$ and $D)$ images show slight hypersignals in the cerebellar WM and the anterior part of the posterior limb of the internal capsule (arrows), indicating myelination.

\section{RESULTS}

\section{Patients}

We added T1-weighted PETRA after routine MR imaging examinations for 76 consecutive patients, of whom 20 were excluded because of severe motion artifacts. Thus, 56 patients (age range, 5 days to 14 years; mean age, 36.6 months; median age, 25 months) were enrolled in this study.

MR imaging was performed for the following reasons: developmental delay $(n=25)$, autism $(n=4)$, epilepsy $(n=4)$, rigidity of the limbs $(n=3)$, small head size $(n=2)$, large head size $(n=$ $1)$, eye-movement disorder $(n=2)$, mental retardation $(n=1)$, headache $(n=1)$, follow-up examination of congenital cytomegalovirus infection $(n=2)$, screening for patients with neurofibromatosis type $1(n=2)$ and café-au-lait spots on the skin $(n=1)$, agenesis of the CC detected on fetal ultrasonography $(n=1)$, phenylketonuria $(n=1)$, hypoxic ischemic injury $(n=1)$, cardiopulmonary resuscitation $(n=1)$, follow-up examination of mitochondrial encephalomyopathy, lactic acidosis and strokelike episodes syndrome $(n=1)$, encephalopathy $(n=1)$, folate receptor $\alpha$ deficiency $(n=1)$, and diabetes insipidus $(n=1)$.

\section{Image Analysis}

The results of the MRIs were as follows: no abnormality $(n=29)$, WM volume loss $(n=6)$, hypogenesis $(n=3)$ and agenesis $(n=$ 1) of the CC, parenchymal atrophy $(n=3)$, small cerebellum $(n=$ $3)$, small cerebellum and brain stem $(n=2)$, cerebellar dysplasia and heterotopia $(n=1)$, patchy T2 prolongation in the WM due to congenital cytomegalovirus infection $(n=2)$, multiple foci of T2 prolongation in patients with neurofibromatosis type $1(n=2)$, medial temporal sclerosis $(n=1)$, small foci of hypoxic change at the watershed area $(n=1)$, subacute infarction of the territory of the left middle cerebral artery $(n=1)$, and ventricular dilation $(n=1)$. The measured T1 value of the brain parenchyma ranged from 1273 to $2618 \mathrm{~ms}$ (mean, $1816 \pm 288 \mathrm{~ms}$ ). Thus, for the designated interval of 1000-1800 ms, we set the first TI at a mean of $1283 \pm 213 \mathrm{~ms}$ for PETRA.

\section{Quantitative Analysis}

The WM-GM contrast was measured in 49 patients older than 7 months of age. The ROI measurements could not be performed at the CC in 4 patients ( 1 case of CC agenesis and 3 cases of CC hypogenesis). The results of the WM-GM contrast for MPRAGE and PETRA are summarized in Table 2. Contrast-to-noise ratios were significantly higher for PETRA than for MPRAGE at all the assessed locations. Contrast ratios were significantly higher for PETRA than for MPRAGE in the anterior part of the posterior limb of the internal capsule and in the genu and splenium of the CC. Contrast ratios were not significantly different between MPRAGE and PETRA in the cerebellar WM.

\section{Qualitative Analysis}

The assessed myelination scores for each reader are summarized in the On-line Table. Myelination at the CC was not assessed in 1 case with CC agenesis and another case with CC hypoplasia. In 1 case with a hypoplastic CC splenium, only the genu was assessed. Myelination of the CC was assessed in 1 case with slight CC hypoplasia.

The mean differences in the PETRA to MPRAGE scores for reader 1 were $-0.07-0.09$; the corresponding values for reader 2 were $-0.16-0.09$ (On-line Table). Interrater correlation for the degree of myelination was substantial or nearly perfect between MPRAGE and PETRA at almost all assessed locations (ICC range, 0.80-1.00; Figs 2-4). The ICC was $<0.01$ for the intrarater correlation of the cerebellar WM rated by reader 2, in which all the cases on PETRA were scored 3, and 51 (91.1\%) cases were scored 3 on MPRAGE (the remaining cases were scored 2). Interrater correlation was almost perfect (ICC range, 0.87-0.99) at most assessed locations. The ICC was also $<0.01$ for the interrater correlation for the cerebellar WM on PETRA, where all the cases on PETRA were scored 3 by reader 2 and $52(92.9 \%)$ cases were scored 3 by reader 1 (with the remainder scored 2).

AJNR Am J Neuroradiol 37:1528-34 Aug 2016 www.ajnr.org 


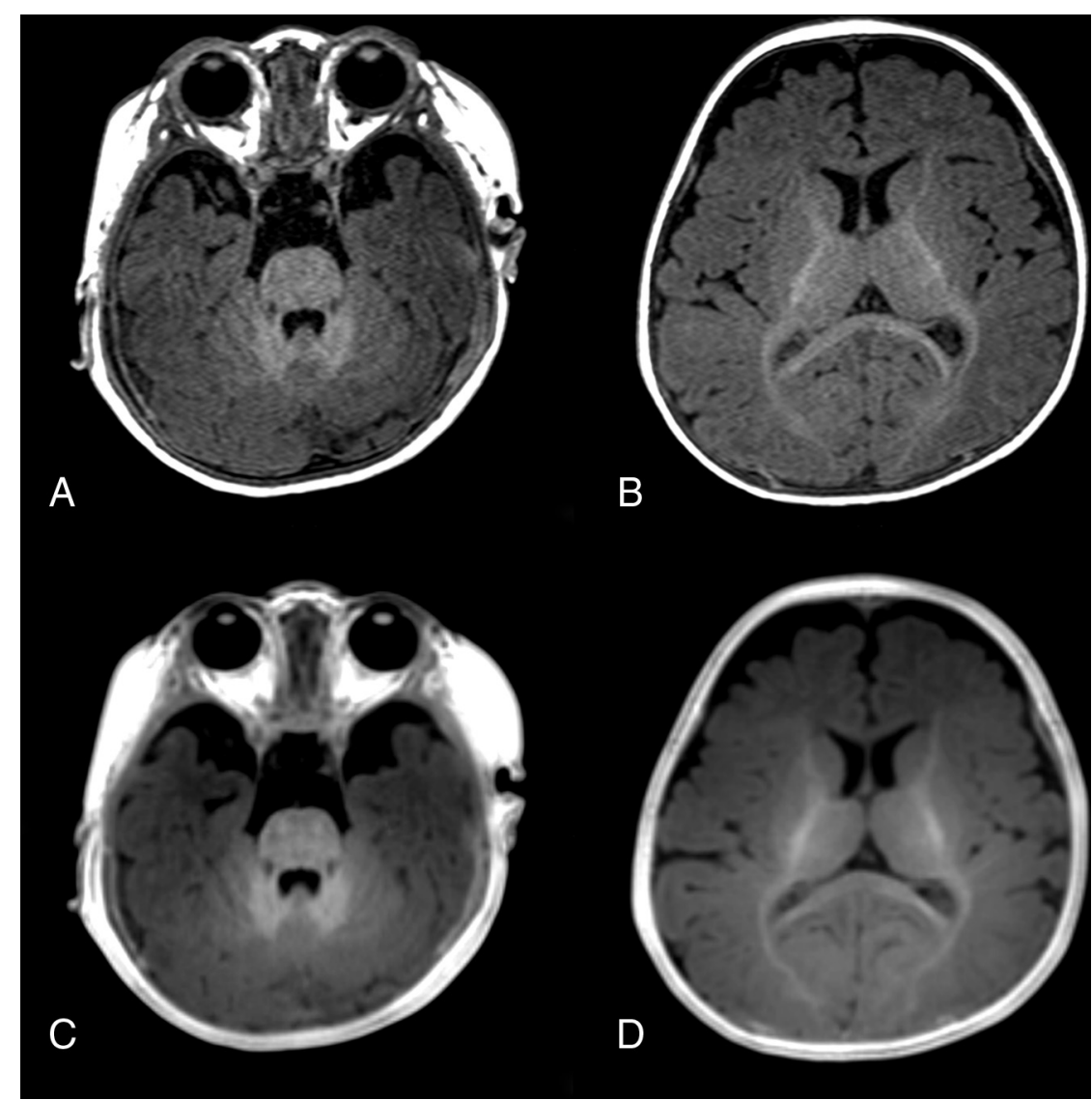

FIG 3. Images of a 5-month-old girl. MPRAGE ( $A$ and $B$ ) and PETRA ( $C$ and $D)$ images both show prominent hypersignals in the cerebellar WM, slight hypersignal in the anterior part of the posterior limb of the internal capsule and the splenium of the corpus callosum, and isosignal in the cerebral cortex at the genu of the corpus callosum and in the subcortical WM of the occipital lobe, indicating myelination.

\section{Acoustic Noise}

The mean acoustic noise levels of MPRAGE and PETRA were 87.4 and $58.2 \mathrm{~dB}(\mathrm{~A})$, respectively, set against an ambient sound level of $53.4 \mathrm{~dB}(\mathrm{~A})$. Notably, the acoustic noise level of PETRA was only $4.8 \mathrm{~dB}(\mathrm{~A})$ higher than that of ambient sound.

\section{DISCUSSION}

We found that PETRA had a generally better WM-GM contrast than MPRAGE. In addition, there were relatively small differences in subjective image quality between MPRAGE and PETRA. Intrarater agreement for MPRAGE and PETRA was substantial or nearly perfect for most of the assessed locations. Although the ICC for the cerebellar WM score on PETRA was very low for reader 2, this result may have been because reader 2 rated all the scores as 3 for cerebellar WM myelination. Actually, small differences were noted in the intrarater and interrater agreement for the cerebellar WM scores. Thus, although further investigation is clearly warranted, these results suggest that there may be no differences in the assessment of myelination between MPRAGE and PETRA.

The assessment of brain myelination as an indicator of brain maturation is one of the main uses of T1WI in pediatrics. ${ }^{16}$ Newer 3T MR imaging scanners yield lower contrast on spin-echo T1WI than $1.5 \mathrm{~T}$ scanners because of $\mathrm{T} 1$ prolongation. ${ }^{17}$ Therefore, gradient-echo sequences such as MPRAGE provide better contrast than conventional spin-echo T1WI. ${ }^{18}$ In addition, volumetric acquisition by using a $3 \mathrm{D}$ gradient-echo sequence provides greater anatomic detail for pathologic assessment than conventional 2D spin-echo T1WI. Indeed, the use of a 3D gradient-echo sequence may play a role as important as T1WI on a 3T MR imaging. ${ }^{19}$ In a previous study of the production of contrast-enhanced images by using PETRA, ${ }^{11}$ equal or better imaging quality was obtained by PETRA compared with MPRAGE for brain tumors. Our results suggest that PETRA also offers quality comparable with that of MPRAGE for noncontrast T1WI of the pediatric brain.

PETRA sequences need to be specified for the pediatric brain. Therefore, we set the first TI on PETRA depending on the individual T1 values, which were measured before PETRA scanning. In fast gradient-echo sequences, longitudinal relaxation of the proton is fully recovered at the first echo, whereas it is partially recovered at subsequent pulses, eventually achieving steadystate. Therefore, several echoes are usually not obtained at first, which is called a "dummy pulse." ${ }^{20}$ With PETRA, however, data are collected from the beginning of the echoes after the first TI to obtain a better signal-to-noise ratio. PETRA acquires data by a pointwise method at the center of $k$-space from the first inversion recovery and from radial projection at the outer edge of the $k$-space after subsequent inversion pulses. Thus, setting the first TI might greatly affect the resulting imaging contrast, so we set the first TI according to individual T1 values. A longer first TI might lead to a better contrast on PETRA, but it means prolongation of the acquisition time. Therefore, we set the first TI to the shortest possible value, preserving the imaging quality for clinical use. The actual setting of the first TI was determined by a preliminary volunteer study. Although further assessment will be needed to determine the appropriate first TI, our results may be appropriate for PETRA T1WI. On the other hand, this process is complex and time-consuming. Therefore, we propose that the first TI be set at $1800 \mathrm{~ms}$ for infants 4 months or younger and $1300 \mathrm{~ms}$ for infants and children older than 4 months of age for clinical use, according to the measured T1 value.

Currently, PETRA has some limitations when setting the sequence. In the current implementation, patients were only scanned with PETRA in the sagittal plane, so section orientation differed between MPRAGE and PETRA. Notably, the parameter setting and spatial resolution differed between PETRA and MPRAGE. In addition, a small FOV cannot be applied for PETRA, and although we set PETRA at a resolution similar to that 


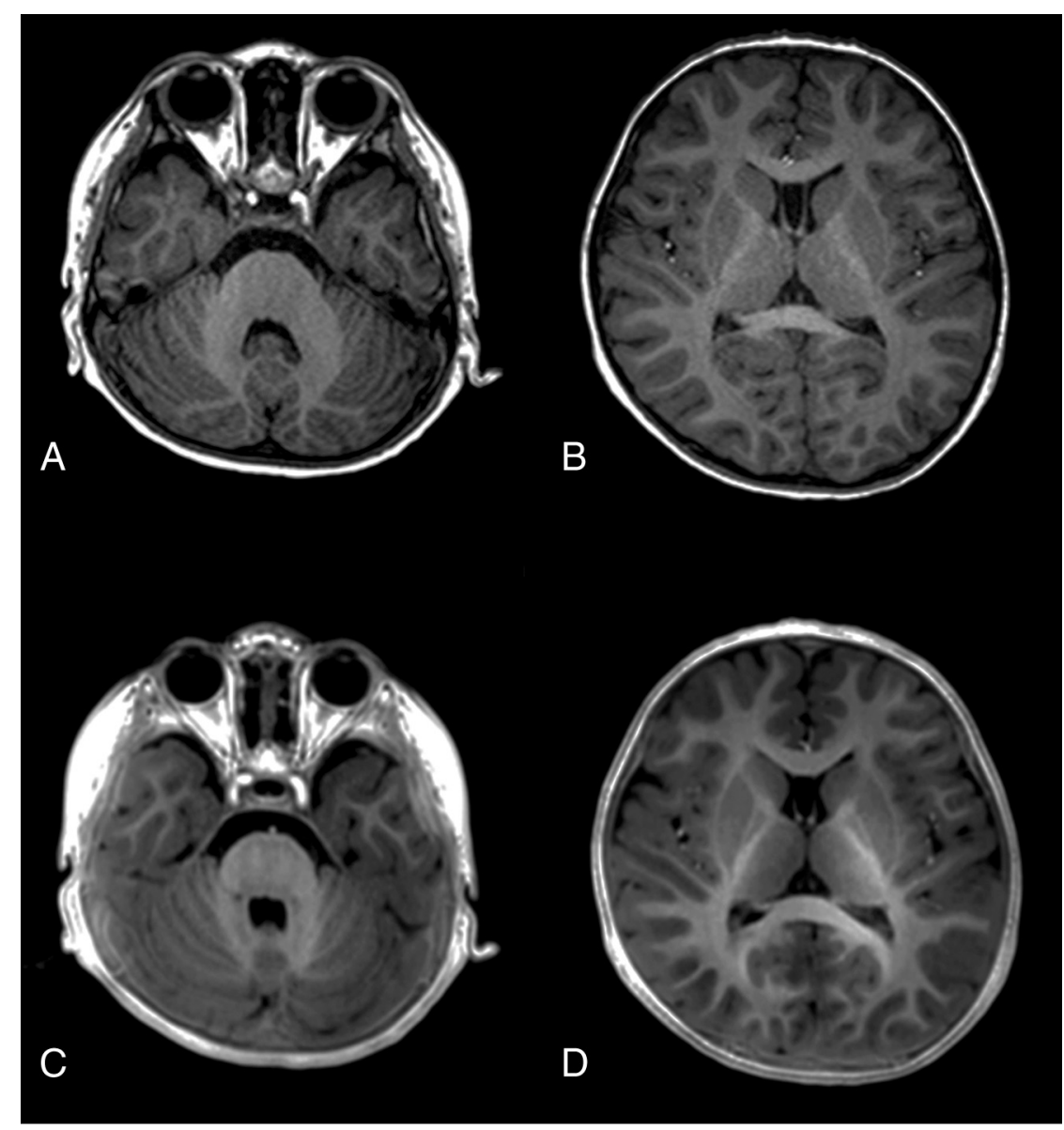

FIG 4. Images of a 27-month-old boy. MPRAGE $(A$ and $B)$ and PETRA ( $C$ and $D)$ images both show prominent hypersignals at all assessed locations, including the cerebellar WM, anterior part of the posterior limb of the internal capsule, genu and splenium of the corpus callosum, and the subcortical WM at the temporal, frontal, and occipital lobes, indicating myelination. should translate to clinical benefit when performing MR imaging examinations in children. Although we measured only a short period for acoustic noise on PETRA, we think that our measurements contained representative noise levels.

The limitations of this study are as follows: First, the sample size was relatively small and comprised a heterogeneous population. The assessed myelination process was, therefore, not based on a normal population. In addition, we only assessed the individual differences between MPRAGE and PETRA, and future studies will be needed to investigate the detectability of intracranial lesions on PETRA, such as ischemic, metabolic, hemorrhagic, and hamartomatous, and some neurodegenerative diseases; those were generally well-visualized on T1WI. We did not assess the utility of PETRA for potential pathologic conditions, but we believe that PETRA could visualize good imaging contrast for neuropathology. On the other hand, detailed morphologic evaluation on PETRA may need more radial spokes for $k$-space data acquisition to avoid image blurring. Although a relatively wide age range was included in this study, we only included patients who needed sedating agents. Consequently, it was a large population of relatively small children. Thus, this can introduce a selection bias. Sec- of MPRAGE, the acquisition time was about 1 minute longer than that of MPRAGE. This might affect the imaging contrast results for PETRA compared with MPRAGE in this study.

Generally, radial acquisition needs a large number of total spokes. However, we used reduced radial spokes on PETRA for clinical use with children to acquire MR data in a relatively short time. However, this use resulted in undersampling for a radial $k$-space acquisition. To improve imaging quality when working with undersampled data, we used 2 techniques before the radial spokes were gridded to the Cartesian grid on PETRA, namely, adaptation of the attenuation matrix to the attenuation present in the Cartesian center of the $k$-space and application of a certain plateau level at the periphery. ${ }^{10,21,22}$ On the other hand, these processes lead to image blurring, which might affect the imaging analysis in this study.

We found that acoustic noise on PETRA was much less than on MPRAGE; this difference was attributed to the unique gradient-pulse sequence of PETRA. PETRA yielded only a slight increase in acoustic noise over background noise, achieving an almost silent sequence. Ida et al ${ }^{11}$ previously measured the acoustic noise of MPRAGE and PETRA by using a clinical 3T MR imaging scanner, which resulted in noise levels of $78.1 \mathrm{~dB}(\mathrm{~A})$ and 51.4 $\mathrm{dB}(\mathrm{A})$, respectively. These results are consistent with our results, indicating negligible acoustic noise when using PETRA, which ond, the qualitative analysis was performed in patients older than 7 months of age because in infants and small children, it was difficult to put the ROI in areas where the myelination showed less progression. Therefore, we only assessed myelination qualitatively for children 7 months of age and younger. In addition, image noise was not assessed because of the differences in the reconstruction methods used for MPRAGE and PETRA.

\section{CONCLUSIONS}

Compared with MPRAGE, the use of PETRA generally achieved better objective imaging quality without a difference in subjective image quality. In addition, PETRA produced near-silent scanning conditions. Therefore, PETRA can substitute for MPRAGE when scanning pediatric patients.

\section{ACKNOWLEDGMENTS}

The authors would like to thank Dr David M. Grodzki, Magnetic Resonance, Siemens AG, Erlangen, Germany, and Mr Yasutake Muramoto, Department of Radiology, Kanagawa Children's Medical Center, for their technical advice.

Disclosures: Noriko Aida_UNRELATED: Grants/Grants Pending: The Japan Society for the Promotion of Science Grants-in-Aid for Scientific Research grant No. 26461843. Katsutoshi Murata-UNRELATED: Employment: Siemens Healthcare K.K. 


\section{REFERENCES}

1. Tocchio S, Kline-Fath B, Kanal E, et al. MRI evaluation and safety in the developing brain. Semin Perinatol 2015;39:73-104 CrossRef Medline

2. Leach JL, Holland SK. Functional MRI in children: clinical and research applications. Pediatr Radiol 2010;40:31-49 CrossRef Medline

3. Arlachov Y, Ganatra RH. Sedation/anaesthesia in paediatric radiology. Br J Radiol 2012;85:e1018-31 CrossRef Medline

4. Slovis TL. Sedation and anesthesia issues in pediatric imaging. Pediatr Radiol 2011;41(suppl 2):514-16 CrossRef Medline

5. Starkey E, Sammons HM. Sedation for radiological imaging. Arch Dis Child Educ Pract Ed 2011;96:101-06 CrossRef Medline

6. Katsunuma A, Takamori H, Sakakura Y, et al. Quiet MRI with novel acoustic noise reduction. MAGMA 2002;13:139-44 CrossRef Medline

7. Segbers M, Rizzo Sierra CV, Duifhuis $\mathrm{H}$, et al. Shaping and timing gradient pulses to reduce MRI acoustic noise. Magn Reson Med 2010;64:546-53 CrossRef Medline

8. Weiger M, Pruessmann KP, Hennel F. MRI with zero echo time: hard versus sweep pulse excitation. Magn Reson Med 2011;66:379-89 CrossRef Medline

9. Idiyatullin D, Corum C, Park JY, et al. Fast and quiet MRI using a swept radiofrequency. J Magn Reson 2006;181:342-49 CrossRef Medline

10. Grodzki DM, Jakob PM, Heismann B. Ultrashort echo time imaging using pointwise encoding time reduction with radial acquisition (PETRA). Magn Reson Med 2012;67:510-18 CrossRef Medline

11. Ida M, Wakayama T, Nielsen ML, et al. Quiet T1-weighted imaging using PETRA: initial clinical evaluation in intracranial tumor patients. J Magn Reson Imaging 2015;41:447-53 CrossRef Medline

12. Dournes G, Grodzki D, Macey J, et al. Quiet submillimeter MR im- aging of the lung is feasible with a PETRA sequence at 1.5 T. Radiology 2015;276:258-65 CrossRef Medline

13. Williams LA, Gelman N, Picot PA, et al. Neonatal brain: regional variability of in vivo $\mathrm{MR}$ imaging relaxation rates at $3.0 \mathrm{~T}$-initial experience. Radiology 2005;235:595-603 CrossRef Medline

14. Gelman N, Ewing JR, Gorell JM, et al. Interregional variation of longitudinal relaxation rates in human brain at $3.0 \mathrm{~T}$ : relation to estimated iron and water contents. Magn Reson Med 2001;45:71-79 Medline

15. Landis JR, Koch GG. The measurement of observer agreement for categorical data. Biometrics 1977;33:159-74 CrossRef Medline

16. Counsell SJ, Maalouf EF, Fletcher AM, et al. MR imaging assessment of myelination in the very preterm brain. AJNR Am J Neuroradiol 2002;23:872-81 Medline

17. Tachibana $Y$, Niwa $T$, Kwee TC, et al. Effective performance of $T(1)$ weighted FLAIR imaging with BLADE in pediatric brains. Magn Reson Med Sci 2012;11:17-26 CrossRef Medline

18. Sarikaya B, McKinney AM, Spilseth B, et al. Comparison of spinecho T1- and T2-weighted and gradient-echo T1-weighted images at $3 \mathrm{~T}$ in evaluating very preterm neonates at term-equivalent age. AJNR Am J Neuroradiol 2013;34:1098-103 CrossRef Medline

19. Tortora D, Panara V, Mattei PA, et al. Comparing 3T T1-weighted sequences in identifying hyperintense punctate lesions in preterm neonates. AJNR Am J Neuroradiol 2015;36:581-86 CrossRef Medline

20. Busse RF, Riederer SJ. Steady-state preparation for spoiled gradient echo imaging. Magn Reson Med 2001;45:653-61 CrossRef Medline

21. Pipe JG, Menon P. Sampling density compensation in MRI: rationale and an iterative numerical solution. Magn Reson Med 1999;41: 179-86 Medline

22. Pipe JG. Reconstructing MR images from undersampled data: dataweighting considerations. Magn Reson Med 2000;43:867-75 Medline 